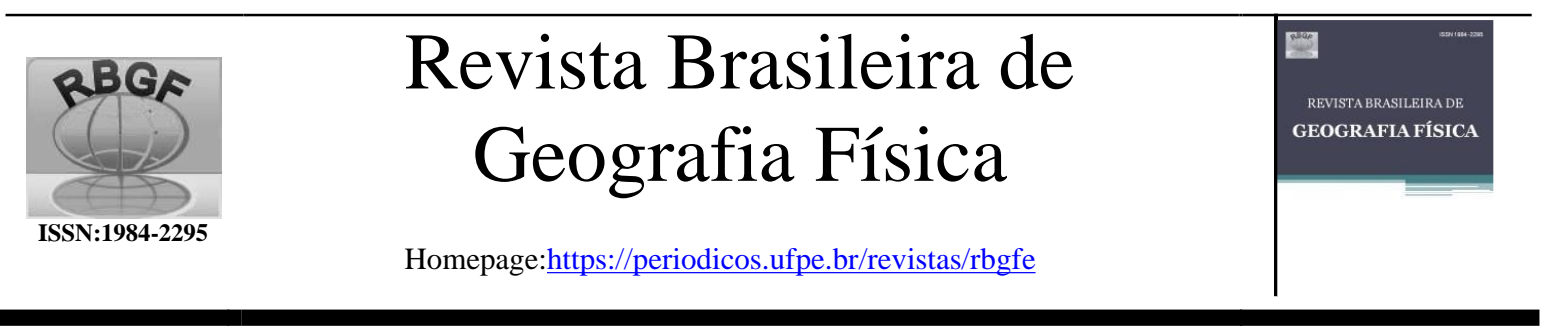

\title{
Qualidade do Solo sob Cultivo de Banana em Sistemas de Produção Orgânico e Convencional em Gonçalves (MG) ${ }^{1}$
}

\author{
Vanêssa Lopes de Faria², Rogério Melloni ${ }^{3}$, Eliane Guimarães Pereira Melloni ${ }^{4}$
}

${ }^{1} \mathrm{O}$ presente artigo é parte da dissertação de mestrado da primeira autora. ${ }^{2}$ Engenheira Ambiental e Sanitária. Mestre em Meio Ambiente e Recursos Hídricos. Universidade Federal de Itajubá (Unifei). Av. BPS, 1303, Bairro Pinheirinho, CEP 37500-903, Itajubá, MG. vanessasgp@yahoo.com.br (autora correspondente). ${ }^{3}$ Professor titular, Instituto de Recursos Naturais, Universidade Federal de Itajubá (Unifei). rmelloni@ unifei.edu.br. ${ }^{4}$ Professora titular, Instituto de Recursos Naturais, Universidade Federal de Itajubá (Unifei). elianegp@unifei.edu.br.

Artigo recebido em 06/04/2020 e aceito em 20/04/2021

\section{R E S U M O}

O objetivo deste trabalho foi avaliar a qualidade do solo em função dos diferentes sistemas de produção orgânico e convencional de banana, mediante análise integrada dos atributos físicos, químicos e microbiológicos, tendo como referência uma área de mata nativa. As amostras deformadas e indeformadas foram coletadas na profundidade de 0 a $20 \mathrm{~cm}$, no município de Gonçalves (MG) e submetidas às análises físicas (textura, densidade do solo, densidade de partículas, porosidade total, diâmetro médio geométrico e ponderado), químicas (fertilidade básica e matéria orgânica), microbiológicas (carbono da biomassa microbiana, atividade microbiana e $\mathrm{qCO}_{2}$ ). Os resultados indicaram que as práticas culturais e o manejo do solo adotado tanto no sistema de produção orgânico quanto no convencional contribuíram para um aporte expressivo de matéria orgânica, melhorando a qualidade do solo quanto aos aspectos físicos, químicos e microbiológicos. Dessa forma, pela semelhança da qualidade do solo, recomenda-se que as práticas de adição de insumos agrícolas nos sistemas de produção convencional sejam revistas.

Palavras-chave: uso do solo, bananicultura, indicadores de qualidade do solo.

\section{Soil Quality under Banana Cultivation in Organic and Conventional Production Systems in Gonçalves (MG)}

\begin{abstract}
The aim of this work was to evaluate the soil quality depending on different banana cultivation conventional and organic systems, through integrated analysis of physical, chemical and microbiological attributes, using an area of a native forest as reference. The deformed and not deformed samples were collected at a depth of 0 to $20 \mathrm{~cm}$ in the city of Gonçalves (Minas Gerais' state), then undergo to physic analysis (texture, soil density, particle density, total porosity, weighted geometric mean diameter); chemical (soil fertility and organic matter); and microbiological (microbial biomass carbon, microbial activity and $q \mathrm{CO}_{2}$ ). The results indicated that the cultural practices and the soil management adopted in the organic and conventional productions system contributed to an expressive input of organic matter, improving soil quality in terms of physical, chemical and microbiological aspects. Thus, due to the similarity of soil quality, it is recommended that the practices of adding agricultural inputs to conventional production systems be reviewed.
\end{abstract}

Keywords: use of the soil, banana cultivation, indicators of the soil quality. 


\section{Introdução}

A preservação e conservação dos recursos naturais, como o solo e a água, remete a um dos grandes desafios da atualidade, visto ser cada vez mais crescente a demanda de alimentos em função, principalmente, do aumento populacional. Segundo informações da Organização das Nações Unidas para a Alimentação e Agricultura (FAO, 2017) estimase que, até 2050, a produção agrícola venha a ser dobrada para suprir a demanda alimentícia, tendo, dessa forma, a intensificação do uso do solo e das áreas produtivas.

Dentre os cultivos em expansão, tem-se o cultivo da bananeira. Este vem ganhando notório destaque, sendo a banana uma das frutas mais produzidas e consumidas em todo o mundo (FAO, 2019). O Brasil, em 2017, ocupou a quarta posição mundial em produção de banana, correspondendo a $5,9 \%$, sendo o estado de Minas Gerais um dos líderes na produção nacional, com destaque para as suas regiões norte e sul (IBGE, 2019). A expansão do cultivo de banana no país é resultante, principalmente, da demanda externa pela fruta, sendo que o aumento da produção ocorre em virtude do avanço das pesquisas agronômicas referentes ao melhoramento genético dos cultivares, controle de doenças e pragas, adubação, espaçamento e outros (Amaro e Fagundes, 2016).

Este aumento denota a importância de avaliar os impactos da produção sobre a qualidade do solo nos sistemas produtivos, como o orgânico e convencional. Quando estes são utilizados de forma ineficiente, contribuem para o desequilíbrio ambiental nas áreas produtivas devido à utilização intensa de agrotóxicos e práticas agrícolas insustentáveis à qualidade do solo, provocando alterações nos seus atributos físicos, químicos e biológicos (Moura Filho et al., 2015; Aires et al., 2019; Amorim et al., 2019). Em contrapartida, sistemas de produção com a adoção de boas práticas propiciam condições adequadas para 0 crescimento e o desenvolvimento das plantas (Araujo et al., 2019; Amorim et al., 2020) e para a manutenção da diversidade de organismos que habitam o solo (Amaro e Fagundes, 2016; Borges et al., 2016). Assim, a adoção de sistemas de produção de alimentos mais eficientes, com menor utilização de recursos ambientais, deve assegurar a produtividade agrícola sem comprometer a capacidade do solo em exercer suas funções no ecossistema e manter a sua qualidade (Schembergue et al., 2017; Assunção et al., 2019).

Considerando o solo como um componente essencial para a expansão agrícola e sustentabilidade ecológica (Alexandre, 2015; Fagundes et al., 2019), surgiu a necessidade de repensar os ambientes por meio da qualidade do solo. Estudos dessa natureza são importantes por fornecerem um conjunto de informações que possibilitam mensurar as alterações provocadas pelos diferentes manejos $\mathrm{e}$ compreender a sustentabilidade dos sistemas de cultivo, fazendo-se necessário o uso de um conjunto mínimo de atributos físicos, químicos e biológicos (Freitas et al., 2017; Li et al., 2019).

Diversos trabalhos têm sido conduzidos com o objetivo de identificar os atributos indicadores de qualidade do solo e seus efeitos no cultivo de banana. Olivares et al. (2020) avaliaram a capacidade produtiva do solo nas duas principais áreas produtoras de banana localizadas na Venezuela (Estados de Aragua e Trujilo). Borges et al. (2018) avaliaram a qualidade de solos irrigados sob cultivo de banana nas regiões Oeste da Bahia e Norte de Minas Gerais baseada nos indicadores físicos, químicos e biológicos considerados mais importantes para o cultivo da banana. Ambos os trabalhos permitiram concluir que os indicadores foram sensíveis e úteis na avaliação da qualidade do solo, constituindo uma ferramenta de suma importância na orientação para os agricultores sobre a seleção das práticas de manejo sustentável do solo.

Diante do exposto e da falta de estudos dos impactos dos cultivos orgânico e convencional da banana no sul de Minas Gerais, o objetivo de presente estudo foi avaliar a qualidade do solo nesses sistemas de produção, mediante análise integrada dos atributos físicos, químicos e microbiológicos, tendo como referência uma área de mata nativa.

\section{Material e métodos}

A escolha do município de Gonçalves (MG) é devida à ampliação da sua produção e da área cultivada de banana nos últimos 15 anos. O município está localizado na região sul do estado de Minas Gerais e completamente inserido na Área de Proteção Ambiental - APA 
Fernão Dias, sendo esta uma Unidade de Conservação Ambiental de Uso Sustentável (UCB, 2013) que tem como finalidade a conciliação da conservação da natureza com o uso sustentável dos recursos naturais (MMA, 2019).

Uma vez definido o município, passouse à escolha das áreas de estudo. Foram selecionadas cinco áreas para avaliação da qualidade do solo (Tabela 1), sendo uma de mata nativa $(\mathrm{MN})$, adotada como área controle e quatro unidades produtivas de banana, caracterizadas por dois diferentes sistemas de produção, sendo dois orgânicos (ORG 1 e ORG 2) e dois convencionais (CONV 1 e CONV 2).

Tabela 1 - Descrição das áreas de estudo em Gonçalves (MG).

\begin{tabular}{c|c|l}
\hline Identificação & Sigla & \multicolumn{1}{c}{ Descrição das áreas } \\
\hline Mata nativa & MN & $\begin{array}{l}\text { Formada por Floresta Ombrófila Mista, onde se destaca a araucária. } \\
\text { Foi utilizada como referência por apresentar um sistema sem } \\
\text { histórico de intervenção humana, sendo observada grande } \\
\text { quantidade de serapilheira e matéria orgânica em decomposição. }\end{array}$ \\
\hline $\begin{array}{c}\text { Sistema de produção } \\
\text { orgânico 1 }\end{array}$ & ORG 1 & $\begin{array}{l}\text { Cultivo de banana instalado sobre antiga mata nativa, há mais de } \\
60 \text { anos, e está há 23 anos sob certificação de conformidade } \\
\text { orgânica. As técnicas de manejo adotadas à época do plantio foram } \\
\text { baseadas em conhecimentos empíricos, nunca sendo realizado } \\
\text { qualquer tipo de adubação, aração, calagem ou necessidade de } \\
\text { controle de pragas e doenças. }\end{array}$ \\
\hline $\begin{array}{c}\text { Sistema de produção } \\
\text { orgânico 2 }\end{array}$ & ORG 2 & $\begin{array}{l}\text { Instalado em antiga área de pastagem, está há 22 anos sob o } \\
\text { certificado de conformidade orgânica. Nunca houve qualquer tipo } \\
\text { de adubação, calagem ou necessidade de controle de pragas e } \\
\text { doenças, sendo as técnicas de manejo adotadas à época do plantio } \\
\text { baseadas em conhecimentos empíricos. }\end{array}$ \\
\hline $\begin{array}{c}\text { Sistema de produção } \\
\text { convencional 1 }\end{array}$ & CONV 1 & $\begin{array}{l}\text { Implantado há 25 anos em antiga área de pastagem, sendo adotadas } \\
\text { técnicas de aração e gradagem à época do plantio. O manejo de } \\
\text { plantas espontâneas é realizado por meio da aplicação de glifosato } \\
N \text {-(phosphonomethyl) glycine, anualmente ou quando necessário. A } \\
\text { correção da acidez do solo é realizada por meio de calcário. }\end{array}$ \\
\hline CONV 2 & $\begin{array}{l}\text { Implantado há 30 anos em antiga área de pastagem, sendo adotadas } \\
\text { técnicas de aração e gradagem à época do plantio. O manejo de } \\
\text { plantas espontâneas é realizado por meio da aplicação de glifosato } \\
N \text {-(phosphonomethyl) glycine, anualmente ou quando necessário. A } \\
\text { correção da acidez do solo é realizada por meio de calcário. }\end{array}$ \\
\hline
\end{tabular}

Ressalta-se que na colheita dos cachos, os sistemas orgânicos e convencionais avaliados depositam sobre o solo cerca de $2 / 3$ da biomassa da bananeira (pseudocaule e folhas), contribuindo para formação da cobertura morta.

$\mathrm{O}$ delineamento experimental adotado foi o de blocos casualizados, em esquema de parcelas, com três repetições. Cinco áreas foram selecionadas e os tratamentos constituídos por dois sistemas de produção de banana orgânicos (ORG 1 e ORG 2); dois sistemas de produção de banana convencionais (CONV 1 e CONV 2) e uma área de mata nativa $(\mathrm{MN})$ adotada como área de referência.

Faria., V., L., Melloni., R., Melloni., E., G., P. 
A coleta das amostras de solo foi realizada em maio de 2019, sendo três amostras deformadas e três indeformadas em cada uma delas. Em cada parcela foi retirada uma amostra composta por três amostras simples de solo, na profundidade de 0 a $20 \mathrm{~cm}$, em ziguezaque e sem adição de serapilheira. Foram analisados os atributos físicos: textura pelo Método da Pipeta; densidade do solo (Ds) e densidade de partículas (Dp) pelo Método do Balão Volumétrico (calculando-se posteriormente a Porosidade Total $(\mathrm{Pt})$ ); estabilidade de agregados em água pelo Método via Úmida (jogo de peneiras na ordem de $2,000 \mathrm{~mm}, 1,000 \mathrm{~mm}, 0,500 \mathrm{~mm}$ e $0,250 \mathrm{~mm}$ e $0,106 \mathrm{~mm}$ de abertura de malha), calculando-se, posteriormente, o diâmetro médio geométrico (DMG) e o diâmetro médio ponderado (DMP).

As amostras indeformadas foram coletadas utilizando-se cilindros metálicos de Koppec (Método do Anel Volumétrico) com auxílio do amostrador de Uhland e, assim, utilizadas para a análise de densidade do solo (Ds). Todas as análises deformadas e indeformadas foram realizadas em triplicatas e seguiram os métodos contidos no manual de Métodos de Análise de Solo da Empresa Brasileira de Pesquisa Agropecuária (Embrapa, 1997), sendo previamente secas ao ar.

Para caracterização química, as amostras deformadas foram peneiradas em malha de $2 \mathrm{~mm}$ de diâmetro e, posteriormente, a realização das análises referentes a: $\mathrm{pH}$ em água na relação de 1:2,5 (solo:água); bases trocáveis: cálcio $(\mathrm{Ca})$, magnésio $(\mathrm{Mg})$ e alumínio $(\mathrm{Al})$ extraídos com $\mathrm{KCl}$ $1 \mathrm{~mol} / \mathrm{L}$, analisados por titulometria; fósforo (P) e potássio (K), extraídos pelo método Mehlich 1 e analisados por colorimetria e fotometria de chama, respectivamente; acidez potencial $(\mathrm{H}+\mathrm{Al})$ extraída pela solução SMP (Schumaker, Mc Lean e Pratney). Fósforo remanescente (P-rem) pelo método do $\mathrm{P}$ em solução de equilíbrio. A determinação da matéria orgânica (MO) foi realizada pelo método proposto por Walkley e Black. A partir dos dados analíticos foram calculadas a Saturação de bases (V\%); Saturação por alumínio (m\%); CTC efetiva (t) e CTC a pH 7,0 (T). Os métodos utilizados encontram-se descritos em Embrapa (1997).

Para as análises microbiológicas, a atividade microbiana foi determinada pelo método da captura do $\mathrm{CO}_{2}$ liberado durante a incubação do solo, sendo capturado em solução de $\mathrm{NaOH} 1$ $\mathrm{mol} / \mathrm{L} \mathrm{e}$, posteriormente, titulada com $\mathrm{HCl} 1 \mathrm{~mol} / \mathrm{L}$, conforme metodologia proposta por Anderson (1982). O carbono da biomassa microbiana (CBM) foi determinado segundo o método da irradiação/incubação descrito por Ferreira et al. (1999), que consiste na irradiação eletromagnética das amostras de solo em forno de micro-ondas, e posterior quantificação de $\mathrm{CO}_{2}$ liberado após incubação por 14 dias a $28^{\circ} \mathrm{C}$. O quociente metabólico $\left(q \mathrm{CO}_{2}\right)$ foi obtido pela razão entre atividade microbiana e a biomassa microbiana (Anderson e Domsch, 1993).

Os resultados dos atributos físicos, químicos e microbiológicos foram submetidos ao teste de normalidade Shapiro-Wilk $(\mathrm{p}<0,05)$ (Shapiro e Wilk, 1965) e, em seguida, à análise de variância (ANOVA) e comparação das médias por Tukey a 5\% de nível de significância, utilizando-se o software estatístico Sisvar®, versão 5.3 (Ferreira, 2011).

\section{Resultados e discussão}

Indicadores físicos de qualidade do solo.

Os sistemas de produção orgânicos e convencionais realizam manejos semelhantes, diferindo apenas na realização de aração e gradagem à época da implantação dos convencionais 1 e 2 , há 25 e 30 anos, respectivamente, e controle de plantas espontâneas por glifosato (Tabela 1). De acordo com a classificação sugerida pelo Manual de Métodos de Análise de Solos (Embrapa, 1997), os solos em estudo pertencem à classe textura média (Tabela 2).

A textura ideal para solos cultivados com bananeira é média ou argilosa por apresentarem melhores condições físicas para o desenvolvimento do plantel, não sendo sugeridos solos com textura arenosa devido à retenção de água e nutrientes (Souza et al., 2016).

Não houve diferença significativa para a densidade do solo (Ds) das áreas de estudo que variaram de $0,8 \mathrm{~g} \mathrm{~cm}^{-3}$ a $1,1 \mathrm{~g} \mathrm{~cm}^{-3}$ (Tabela 2). Quanto mais elevada a Ds, maiores serão as restrições para o crescimento e desenvolvimento radicular das plantas (Mioto et al., 2020), uma vez que o deslocamento de água e ar para a rizosfera das plantas ocorre pelo espaço poroso (Jesus et al., 2017). Dessa forma, pode-se considerar que, quanto maior a quantidade de espaços porosos, maior a infiltração de água e desenvolvimento radicular.

A MN, utilizada como referência, apresentou valor semelhante de Ds comparativamente aos sistemas orgânicos e convencionais, evidenciando que o uso antrópico não interferiu na compactação do solo. Com exceção da área $\mathrm{ORG} 1$, que anteriormente à 
implantação do sistema de produção havia uma mata nativa e considerando que as áreas ORG 2, CONV 1 e CONV 2 eram anteriormente utilizadas como área de pastagem e recebiam constantes pressões sobre o solo advindos de pisoteio animal e baixa deposição de matéria orgânica (Tabela 1), verifica-se que o sistema de manejo adotado para o cultivo de banana proporcionou melhorias na qualidade física do solo. Sendo assim, os resultados de Ds podem ser atribuídos ao constante aporte de matéria orgânica pela cobertura vegetal nos

Tabela 2 - Atributos físicos do solo nas diferentes áreas de estudo em Gonçalves (MG).

\begin{tabular}{|c|c|c|c|c|c|c|c|c|c|}
\hline Áreas & Argila & $\begin{array}{l}\text { Areia } \\
--\%--\end{array}$ & Silte & $\begin{array}{l}\text { Classe } \\
\text { Textural }\end{array}$ & $\begin{array}{l}\text { Ds } \\
----\mathrm{g} \mathrm{cm}^{-}\end{array}$ & $\begin{array}{c}\mathrm{Dp} \\
\mathrm{n}^{-3}-\mathrm{-}^{--}\end{array}$ & $\begin{array}{l}\mathrm{Pt} \\
\%\end{array}$ & $\begin{array}{l}\text { DMG } \\
-----m\end{array}$ & $\begin{array}{l}\text { DMP } \\
\text { m----- }\end{array}$ \\
\hline MN & $36,8 \mathrm{a}$ & $40,7 \mathrm{a}$ & $22,4 a$ & Média & $0,8 \mathrm{a}$ & $2,2 \mathrm{a}$ & $63,6 a$ & $3,7 \mathrm{a}$ & $4,5 \mathrm{a}$ \\
\hline ORG 1 & $32,3 a$ & $53,0 \mathrm{a}$ & $14,7 \mathrm{c}$ & Média & $1,0 \mathrm{a}$ & $2,3 \mathrm{a}$ & $56,5 \mathrm{a}$ & $4,0 \mathrm{a}$ & $6,4 a$ \\
\hline ORG 2 & $24,4 a b$ & $53,3 a$ & $22,3 b c$ & Média & $1,1 \mathrm{a}$ & $2,3 \mathrm{a}$ & $52,2 \mathrm{a}$ & $3,9 a$ & $4,6 a$ \\
\hline CONV 1 & $12,3 \mathrm{~b}$ & $42,9 a$ & $44,9 \mathrm{a}$ & Média & $0,9 \mathrm{a}$ & $2,2 \mathrm{a}$ & $59,1 \mathrm{a}$ & $3,8 \mathrm{a}$ & $6,7 \mathrm{a}$ \\
\hline CONV 2 & $14,1 \mathrm{~b}$ & $48,8 \mathrm{a}$ & $37,2 \mathrm{ab}$ & Média & $0,9 a$ & $2,2 \mathrm{a}$ & $59,1 \mathrm{a}$ & $4,1 \mathrm{a}$ & $4,7 \mathrm{a}$ \\
\hline CV (\%) & 10,55 & 22,92 & 21,50 & --------- & 12,68 & 3,27 & $12 \%$ & 6,21 & 17,68 \\
\hline
\end{tabular}

Áreas de estudo: mata nativa (MN), sistemas de produção orgânicos (ORG 1, ORG 2), sistema de produção convencionais (CONV 1, CONV 2). Coeficiente de variação (CV). Valores médios de porcentagem de argila, areia, silte, densidade do solo (Ds), densidade de partículas (Dp), porosidade total $(\mathrm{Pt})$, diâmetro médio geométrico (DMG) e diâmetro médio ponderado (DMP) na profundidade de 0-20 cm. Médias seguidas de mesma letra na coluna não diferem entre si pelo Teste de Tukey a 5\% de significância.

Diferentemente dos valores de Ds encontrados neste trabalho, Aires et al. (2019) verificaram valores superiores de Ds em áreas de cultivo anual e perene de banana sob manejo convencional no Vale Submédio do São Francisco, Bahia, comparativamente ao manejo orgânico. Segundo os autores, estes valores elevados foram resultantes do manejo realizado no solo que é baseado na aração e gradagem, resultando no revolvimento da camada superficial do solo e ocasionando, dessa forma, pressão no solo das máquinas agrícolas e diminuição da Ds.

Corroborando os dados para Ds encontrados neste estudo, Pereira Junior et al. (2018) também observaram valores semelhantes de Ds em áreas sob o cultivo de banana orgânica e convencional no Sertão Paraibano, não apresentando valores considerados impeditivos ao bom desenvolvimento radicular das plantas.

Os valores de Dp pouco variaram (2,2 a $\left.2,3 \mathrm{~g} \mathrm{~cm}^{-3}\right)$, sem diferença significativa entre as áreas (Tabela 2). Esta pouca variação, de acordo com Balota (2018), pode estar relacionada à composição mineralógica e ao conteúdo de matéria orgânica, não sendo influenciada por alterações do manejo. Os componentes que predominam em solos minerais apresentam valores de Dp que variam, em média, entre os limites de 2,3 e $2,9 \mathrm{~g} \mathrm{~cm}^{-3}$, exceto quando na presença de altos teores de matéria orgânica, quando a Dp diminui, ou óxidos de $\mathrm{Fe}$ e $\mathrm{Al}$ quando a Dp aumenta (Aires et al., 2019). Os valores para $\mathrm{Dp}$ observados neste trabalho foram inferiores a $2,3 \mathrm{~g} \mathrm{~cm}^{-3}$ para todos os cultivos, existindo, possivelmente, influência da matéria orgânica nesses solos.

Valores de Dp inferiores a $2,3 \mathrm{~g} \mathrm{~cm}^{-3}$ também foram relatados por Aires et al. (2019) em sistemas de cultivo orgânico e convencional de banana. Os autores justificaram a baixa Dp devido à influência da matéria orgânica também observada nos solos daquele estudo.

A maior mobilização do solo pela movimentação de máquinas e implementos agrícolas pode reduzir a $\mathrm{Pt}$ e modificar a distribuição de poros por tamanho. Valores de Pt reduzidos indicam alterações na dinâmica de água e gases, menor capacidade de armazenamento de água o solo e na infiltração, bem como na disponibilidade de água para as 
culturas (Fagundes et al., 2019. Na avaliação do atributo $\mathrm{Pt}$, não foi observada diferença estatística significativa entre as áreas, sendo que os valores se apresentaram entre $52,2 \%$ para o sistema ORG 2 e $59,1 \%$ para os sistemas convencionais CONV 1 e CONV 2, sem diferença com a área de MN (Tabela 2).

Estes dados foram contrários aos observados por Ribeiro et al. (2018) quando estudaram as condições físicas de Chernossolos cultivados com outra cultura, soja, na bacia hidrográfica do Rio Santa Maria onde houve condições limitantes para o desenvolvimento das raízes para a variável Pt, comparativamente à área de mata nativa. Os autores concluíram que o preparo convencional proporcionou condições físicas inadequadas para o pleno desenvolvimento da soja devido, principalmente, à drenagem e à aeração deficientes dos solos nestas áreas. A análise da estabilidade dos agregados é de suma importância na avaliação do solo, pois está relacionada a importantes processos, como manejo ou distúrbios, sendo capazes de impactar sobre a MO e estabilidade geral do solo a processos erosivos (Sales et al., 2016; Loss et al., 2017). Não houve diferença significa entre as áreas de estudo para os indicadores DMG e DMP, que variaram de 3,7 a $4,1 \mathrm{~mm}$ e de 4,5 a 6,7 mm, respectivamente. Os resultados evidenciam que a ausência de revolvimento do solo pelo tráfego de maquinários e implementos agrícolas, aliados à maior cobertura do solo pela biomassa da bananeira, preservaram a estrutura dos agregados, implicando em solos bem estruturados (Borges et al., 2018), até mesmo comparados ao da MN (Tabela 2).

Os dados neste estudo corroboram aqueles obtidos por Rosa et al. (2019), em outras condições de uso do solo, quando concluíram que a estabilidade dos agregados não diferiu significativamente em áreas sob cultivo de eucalipto/pastagem e uma área de cerrado nativo.

Rossi et al. (2016) avaliaram a formação de agregados em sistema de manejo agroecológico formado, dentre outros, por banana, e observaram que as áreas com maior cobertura do solo favoreceram a lenta decomposição da matéria orgânica, o que contribuiu para uma maior estabilidade dos agregados e, consequentemente, aumento nos valores de DMG e DMP, corroborando os dados obtidos no presente trabalho em relação à estabilidade do solo das áreas estudadas.

Estudo conduzido por Sales et al. (2016), em outra condição de uso do solo, revelou maior conteúdo de carbono orgânico total em sistema de plantio direto quando comparado ao sistema de preparo convencional em Nova Porteirinha (MG). Segundo os autores, a ausência de revolvimento no solo, associada a manejos como o pousio da área anterior à implantação do cultivo, presença de plantas espontâneas e cobertura morta produzida pelas sucessões favoreceram maior alocação de carbono nas camadas superficiais do solo.

A importância do manejo na alteração dos atributos físicos do solo fica comprovada nos estudos realizados por Prakash et al. (2019) e Almeida et al. (2019), os quais, estudando a cultura de banana, concluíram que a adoção de sistemas conservacionistas melhora a qualidade física do solo, o crescimento, a produção e a qualidade dos frutos. Com base nos resultados dos indicadores físicos de qualidade do solo, as práticas culturais e o manejo do solo adotados nos sistemas de produção orgânico e convencional de Gonçalves (MG) contribuíram para um maior aporte de matéria orgânica, evidenciando, portanto, benefícios à qualidade física do solo.

Indicadores químicos de qualidade do solo

As médias dos indicadores químicos $\mathrm{pH}, \mathrm{K}, \mathrm{P}, \mathrm{Ca}^{2+}, \mathrm{Mg}^{2+}, \mathrm{Al}^{3+}, \mathrm{H}+\mathrm{Al}, \mathrm{SB}, \mathrm{CTC}$ efetiva, $\mathrm{CTC}_{\mathrm{pH}} 7,0$, saturação por bases, saturação por alumínio, MO e P remanescente, avaliados na profundidade de $0-20 \mathrm{~cm}$ da mata nativa $(\mathrm{MN})$ e dos sistemas de produção (ORG 1, ORG 2, CONV 1, CONV 2) são apresentadas na Tabela 3.

Não houve diferença significativa para os atributos $\mathrm{P}, \mathrm{CTC}_{\mathrm{pH}} 7,0, \mathrm{MO}$ e $\mathrm{P}$ nas áreas de estudo (Tabela 3). No entanto, verifica-se que os teores de $\mathrm{P}$ encontrados nas áreas de estudo variaram de 4,0 $\mathrm{mg} \mathrm{dm}^{-3}$ na MN e $8,2 \mathrm{mg} \mathrm{dm}^{-3}$ em CONV 2, valores estes considerados inferiores aos adequados para a cultura de banana que, segundo Borges et al. (2016) seriam de $16 \mathrm{mg} \mathrm{dm}^{-3}$ a $30 \mathrm{mg} \mathrm{dm}^{-3}$. Os mesmos autores relatam que a deficiência de $P$ proporciona coloração verde-escura tendendo a azulada das folhas mais velhas e posterior necrose, além de menor teor de açúcar, 
dificultando a sua comercialização. Entretanto, para as áreas estudadas não se observaram sintomas de deficiência de $\mathrm{P}$, apesar dos baixos índices, fato este devido ao alto teor de MO que tem potencial para mineralizar grande quantidade de $\mathrm{P}$ e reduzir a adsorção do $\mathrm{P}$ remanescente (Amorim et al., 2019).

Tabela 3 - Valores médios dos indicadores químicos avaliados na profundidade 0-20 $\mathrm{cm}$ da Mata Nativa (MN) e dos Sistemas de Produção (ORG 1, ORG 2, CONV 1, CONV 2)

\begin{tabular}{|c|c|c|c|c|c|c|}
\hline \multirow{3}{*}{ Indicador } & \multirow{3}{*}{$\mathrm{CV}(\%)$} & \multirow{3}{*}{ MN } & \multicolumn{4}{|c|}{ Sistema de Produção } \\
\hline & & & \multicolumn{2}{|c|}{ ORG } & \multicolumn{2}{|c|}{ CONV } \\
\hline & & & 1 & 2 & 1 & 2 \\
\hline $\mathrm{pH}\left(\mathrm{H}_{2} \mathrm{O}\right)$ & 5,21 & $4,6 \mathrm{~b}$ & $4,7 b$ & $5,2 \mathrm{~b}$ & $6,2 \mathrm{a}$ & $6,0 \mathrm{a}$ \\
\hline $\mathrm{K}\left(\mathrm{cmol}_{\mathrm{c}} \mathrm{dm}^{-3}\right)$ & 18,58 & $0,20 \mathrm{ab}$ & $0,22 \mathrm{ab}$ & $0,14 \mathrm{~b}$ & $0,34 \mathrm{ab}$ & $0,43 \mathrm{a}$ \\
\hline$P\left(\mathrm{mg} \mathrm{dm}^{-3}\right)$ & 43,28 & $4,0 \mathrm{a}$ & $5,7 \mathrm{a}$ & $6,4 a$ & $6,8 \mathrm{a}$ & $8,2 \mathrm{a}$ \\
\hline $\mathrm{Ca}^{2+}\left(\mathrm{cmol}_{\mathrm{c}} \mathrm{dm}^{-3}\right)$ & 42,55 & $0,8 \mathrm{c}$ & $2,5 b c$ & $2,3 c$ & $9,1 \mathrm{a}$ & $7,2 \mathrm{ab}$ \\
\hline $\mathrm{Mg}^{2+}\left(\mathrm{cmol}_{\mathrm{c}} \mathrm{dm}^{-3}\right)$ & 55,86 & $0,4 \mathrm{~b}$ & $0,9 \mathrm{ab}$ & $0,9 \mathrm{ab}$ & $2,9 a$ & $2,1 \mathrm{a}$ \\
\hline $\mathrm{Al}^{3+}\left(\mathrm{cmol}_{\mathrm{c}} \mathrm{dm}^{-3}\right)$ & 48,02 & $1,6 \mathrm{c}$ & $0,9 b c$ & $0,5 \mathrm{ab}$ & $0,1 \mathrm{a}$ & $0,1 \mathrm{a}$ \\
\hline $\mathrm{H}+\mathrm{Al}\left(\mathrm{cmol}_{\mathrm{c}} \mathrm{dm}^{-3}\right)$ & 27,81 & $11,7 \mathrm{~b}$ & $10,0 \mathrm{~b}$ & $6,7 \mathrm{ab}$ & $3,8 \mathrm{a}$ & $3,1 \mathrm{a}$ \\
\hline $\mathrm{SB}\left(\mathrm{cmol}_{\mathrm{c}} \mathrm{dm}^{-3}\right)$ & 41,97 & $1,4 \mathrm{c}$ & $3,7 \mathrm{bc}$ & $3,4 \mathrm{bc}$ & $12,4 \mathrm{a}$ & $9,8 \mathrm{ab}$ \\
\hline CTC efetiva $\left(\mathrm{cmol}_{\mathrm{c}} \mathrm{dm}^{-3}\right)$ & 36,88 & $3,0 \mathrm{c}$ & $4,6 b c$ & $3,9 \mathrm{c}$ & $12,4 \mathrm{a}$ & $9,9 \mathrm{ab}$ \\
\hline $\mathrm{CTC}_{\mathrm{pH} 7,0}\left(\mathrm{cmol}_{\mathrm{c}} \mathrm{dm}^{-3}\right)$ & 19,06 & $12,8 \mathrm{a}$ & $13,7 \mathrm{a}$ & $10,1 \mathrm{a}$ & $16,1 \mathrm{a}$ & $13,0 \mathrm{a}$ \\
\hline Saturação por Bases $(\%)$ & 24,70 & $10,9 b$ & $27,8 \mathrm{~b}$ & $33,9 b$ & $74,7 \mathrm{a}$ & $74,7 \mathrm{a}$ \\
\hline Saturação por Alumínio (\%) & 66,92 & $54,9 \mathrm{c}$ & $21,2 b c$ & $14,3 \mathrm{ab}$ & $0,5 \mathrm{a}$ & $0,9 \mathrm{a}$ \\
\hline $\mathrm{MO}\left(\mathrm{dag} \mathrm{kg}^{-1}\right)$ & 27,99 & $4,0 \mathrm{a}$ & $3,6 \mathrm{a}$ & $3,1 \mathrm{a}$ & $4,9 \mathrm{a}$ & $3,2 \mathrm{a}$ \\
\hline$P$ remanescente $\left(\mathrm{mg} \mathrm{L}^{-1}\right)$ & 27,17 & $13,3 \mathrm{a}$ & $18,7 \mathrm{a}$ & $21,9 \mathrm{a}$ & $21,4 \mathrm{a}$ & $24,3 \mathrm{a}$ \\
\hline
\end{tabular}

Áreas de estudo: MN (Mata Nativa) e Sistemas de Produção (ORG 1, ORG 2, CONV 1 e CONV 2). Coeficiente de variação (CV \%). Médias seguidas de mesma letra na linha não diferem entre si pelo Teste de Tukey a 5\% de significância

Visto que não houve diferença significativa para a MO, os valores encontrados nos sistemas de produção podem estar relacionados ao constante aporte de matéria orgânica (Assunção et al., 2019; Li et al., 2019; Yang et al., 2019; Barbosa et al., 2020), como a biomassa da bananeira, uma vez que, no corte dos cachos, o pseudocaule é deixado sobre o solo, juntamente com as folhas, frutos e engaço (parte que sustenta o cacho da fruta). Ressaltase que esta prática é realizada em todos os sistemas de produção, orgânicos e convencionais avaliados, e à constante deposição de serapilheira na MN. Posto que as áreas ORG 2, CONV 1 e CONV 2 anteriormente à implantação do plantel foram utilizadas como área de pastagem sem qualquer manejo (Tabela 1), pode-se inferir que, possivelmente, a entrada de carbono orgânico via deposição de biomassa de bananeira foi suficiente para elevar o teor de MO ao mesmo nível do sistema ORG 1 e MN (Tabela 3). Melo et al. (2017) também observaram valores semelhantes de MO em áreas sob cultivo de cana-de-açúcar (em sucessão ao cultivo de hortaliças com elevado aporte de estercos) e cultivo consorciado de milho, feijão-caupi e mandioca em área anteriormente utilizada por plantação de banana. Para os autores, os manejos adotados nestas áreas indicam que a adoção das práticas realizadas favoreceu a manutenção e o incremento de MO no solo, ressaltando a importância que desempenham em todos os compartimentos do solo. Silva et al. (2007) avaliaram a qualidade do solo cultivado com banana Prata Anã no Norte de Minas Gerais e observaram que os solos com maior produtividade foram aqueles com maiores valores de argila, silte, capacidade de troca de catiônica (CTC), saturação por base (V \%) e $\mathrm{MO}$, valores estes que corroboram os deste estudo. 
Os indicadores químicos $\mathrm{pH}, \mathrm{K}, \mathrm{Ca}^{2+}$, $\mathrm{Mg}^{2+}, \mathrm{Al}^{3+}, \mathrm{H}+\mathrm{Al}, \mathrm{SB}, \mathrm{CTC}$ efetiva, V\% e saturação por Alumínio apresentaram diferença significativa nas áreas cultivadas. Os valores de pH variaram de 4,7 a 6,2 apresentando caráter ácido. Entretanto, a MN apresentou o mesmo comportamento, valor de $\mathrm{pH}$ abaixo da neutralidade, com $\mathrm{pH}$ médio de 4,6. Borges et al. (2016) descrevem que o $\mathrm{pH}$ recomendado para o cultivo de banana deve variar de 6,0 a 6,5. Os sistemas convencionais CONV $1 \mathrm{e}$ CONV 2 possuem uma acidez baixa tendendo à neutralidade, com pH variando de 6,0 a 6,2, possuindo, portanto, valores de $\mathrm{pH}$ recomendados, em virtude de ações antrópicas de correção do solo por meio de calagem (Borges e Souza, 2009).

Benefícios na fauna edáfica do solo foram relacionados à calagem, com consequente elevação do $\mathrm{pH}$, somada à cobertura do solo em áreas de bananais por Cesaril et al. (2019). Segundo os autores, a calagem nos bananais proporcionou melhorias na qualidade química, física e biológica do solo decorrentes desta prática, consequentemente, favorecendo os grupos Acari, Araneae e Collembola, decompositores e envolvidos na ciclagem e mineralização da MO.

Os sistemas de produção CONV 1 e CONV 2 apresentaram valores de saturação por base equivalentes a 74,7\%. Da mesma forma, a calagem realizada nos sistemas convencionais resultou em aumento na SB, corroborando o estudo de Oliveira et al. (2021), quando avaliaram os atributos químicos do solo sob cultivo de banana irrigada, na Região do Vale do São Francisco.

A calagem, prática adotada nas áreas CONV 1 e CONV 2, além de elevar o pH do solo, contribui para aumentar a disponibilidade de $\mathrm{K}$, neutralizar o $\mathrm{Al}^{3+}$, fornecer $\mathrm{Ca}^{2+}{\mathrm{e} \mathrm{Mg}^{2+}}^{2+}$ para as plantas e, consequentemente, elevar a saturação por bases, condizendo com os valores encontrados neste estudo (Borges et al., 2016). $\mathrm{A}$ ausência de $\mathrm{Al}^{3+}$ relaciona-se com o $\mathrm{pH}$ adequado do solo, ou seja, o $\mathrm{Al}^{3+}$ é reduzido à medida que o $\mathrm{pH}$ aumenta. Dessa forma, a MN e o sistema ORG 1 apresentaram valores elevados de $\mathrm{Al}^{3+}$, seguido pelo valor médio de $0,5 \mathrm{cmol}_{\mathrm{c}} \mathrm{dm}^{-3}$ no sistema ORG 1 e tendendo à ausência nos sistemas convencionais CONV $1 \mathrm{e}$ CONV 2. No entanto, mesmo com valores mais altos de $\mathrm{Al}^{+3}$ que as áreas com calagem (CONV
1 e CONV 2), as plantas se desenvolvem e produzem material vegetal que, ao ser depositado no solo, forma a serapilheira necessária para presença de grupos da fauna edáfica (Cesaril et al., 2019) e, consequente mineralização da MO.

Melo et al. (2017) também relacionaram os resultados encontrados para $\mathrm{Ca}^{2+}, \mathrm{Mg}^{2+} \mathrm{e} \mathrm{Al}^{3+}$ à ação da calagem realizada nas áreas cultiváveis. Os teores de $\mathrm{Al}^{3+}$ encontrados por Freitas et al. (2017) também variaram significativamente entre as áreas de estudo, sendo os maiores valores observados em área de mata e os menores em áreas cultiváveis, acompanhando as variações de $\mathrm{pH}$.

Os teores de $\mathrm{Ca}^{2+}$ e $\mathrm{Mg}^{2+}$ foram considerados altos para a cultura de banana nas áreas convencionais CONV 1 e CONV 2, em função da calagem realizada nessas áreas, uma vez que os valores de referência adequados variam 2,5 a $4,8 \mathrm{cmol}_{\mathrm{c}} \mathrm{dm}^{-3}$ e 0,6 a $1,2 \mathrm{cmol}_{\mathrm{c}}$ $\mathrm{dm}^{-3}$, respectivamente (Borges et al., 2016). Tem-se, que os sistemas ORG 1 e ORG 2 apresentaram valores considerados adequados para a cultura, um indicativo de que o manejo realizado nestas áreas pode ser considerado suficiente para a cultura de banana, não havendo, portanto, a necessidade de adição de insumos químicos. Amorim et al. (2019) confirmaram também, em seu estudo, que a incorporação ao solo de restos culturais da bananeira promoveu incremento de nutrientes, principalmente de $\mathrm{Mg}^{2+}$.Os níveis de $\mathrm{K}$ diferiram estatisticamente, sendo que os sistemas ORG 1 e ORG 2 apresentaram valores de $0,14 \mathrm{cmol}_{\mathrm{c}} \mathrm{dm}^{-3}$ e $0,22 \mathrm{cmol}_{\mathrm{c}} \mathrm{dm}^{-3}$, enquanto os recomendados para a cultura de banana variam de 0,31 a $0,60 \mathrm{cmol}_{\mathrm{c}} \mathrm{dm}^{-3}$ (Borges et al., 2016). Dessa forma, os valores de $K$ apresentados nos sistemas CONV 1 e CONV 2 estão dentro das recomendações, enquanto dos sistemas orgânicos ficou aquém do recomendado. Considerando que o $\mathrm{K}$ é o nutriente absorvido em maior quantidade pela bananeira, representando cerca de $37 \%$ distribuído pelo pseudocaule, seguido dos frutos, folhas e engaço, sua deficiência limita o desenvolvimento dos cachos e frutos, além da maturação irregular e baixos teores de sabor (Borges et al., 2016). Os valores obtidos neste estudo para $\mathrm{K}$ nos sistemas convencionais CONV 1 e CONV 2 diferem-se dos observados por Morais et al. (2014) quando verificaram 
redução de $\mathrm{K}$ em áreas cultivadas de bananeira em comparação à área de referência, afirmando que os teores desse elemento decresceram com o uso em virtude das elevadas exigências pela cultura.

Os valores de $\mathrm{H}+\mathrm{Al}$ também variaram entre os sistemas, apresentando comportamento similar ao $\mathrm{Al}^{3+}$ e saturação por alumínio, com maiores valores observados na $\mathrm{MN}$ e menores nos sistemas CONV 1 e CONV 2, corroborando o estudo realizado por Borges et al. (2016) que relacionaram tais resultados ao manejo dos restos culturais, os quais são deixados na superfície do solo.

Em um contexto geral, os resultados permitem inferir que as práticas culturais e o manejo do solo adotado no sistema de produção orgânico e convencional contribuíram para um aporte expressivo de matéria orgânica atribuído à constante deposição da biomassa da bananeira, sem diferença com a área de referência de mata nativa (Tabela 3 ).

Os sistemas de produção CONV 1 e CONV 2 apresentaram resultados adequados para a cultura de banana, onde, além do material orgânico depositado, houve adição de insumos químicos. No entanto, destaca-se que os resultados para os indicadores químicos nos sistemas orgânicos apresentaram condições químicas favoráveis ao desenvolvimento do plantel, ou seja, não haveria mesmo a necessidade de adição ou interferência humana para o cultivo. Diante disto, questiona-se a real necessidade de adição de insumos químicos nos sistemas convencionais adotados para banana, em Gonçalves (MG).

Indicadores microbiológicos de qualidade do solo

As médias dos indicadores microbiológicos (atividade microbiana, carbono da biomassa microbiana e $q \mathrm{CO} 2$ ), avaliados na profundidade de $0-20 \mathrm{~cm}$ da Mata Nativa (MN) e dos Sistemas de Produção (ORG 1, ORG 2, CONV 1, CONV 2) são apresentadas na Tabela 4.

Tabela 4 - Atividade microbiana, carbono da biomassa microbiana e quociente metabólico das áreas de estudo MN (Mata Nativa) e Sistemas de Produção (ORG 1, ORG 2, CONV 1 e CONV 2)

\begin{tabular}{|c|c|c|c|}
\hline \multirow{3}{*}{ Áreas de estudo } & \multicolumn{3}{|c|}{ Indicadores Microbiológicos } \\
\hline & $\begin{array}{l}\text { Atividade } \\
\text { Microbiana }\end{array}$ & $\begin{array}{c}\text { Carbono da Biomassa } \\
\text { Microbiana }\end{array}$ & $q \mathrm{CO}_{2}$ \\
\hline & $\begin{array}{c}\mu \mathrm{g} \mathrm{CO} 2 / \mathrm{g} \text { de solo } \\
\text { seco.dia }\end{array}$ & $\begin{array}{l}\mu \mathrm{g} \mathrm{C} / \mathrm{g} \mathrm{de} \mathrm{C.} \mathrm{g} \mathrm{de} \\
\text { solo seco }\end{array}$ & $\begin{array}{c}\mu \mathrm{gCO} 2 / \mu \mathrm{g} \mathrm{C} . \mathrm{g} \mathrm{de} \\
\text { solo seco. dia }\end{array}$ \\
\hline MN & $68,92 \mathrm{a}$ & $811,57 \mathrm{a}$ & $3,66 \mathrm{a}$ \\
\hline ORG 1 & $38,20 \mathrm{a}$ & $677,13 \mathrm{a}$ & $2,35 \mathrm{a}$ \\
\hline ORG 2 & $71,16 a$ & $927,69 \mathrm{a}$ & $1,79 \mathrm{a}$ \\
\hline CONV 1 & $41,41 \mathrm{a}$ & $705,12 \mathrm{a}$ & $3,79 \mathrm{a}$ \\
\hline CONV 2 & $41,15 \mathrm{a}$ & $815,41 \mathrm{a}$ & $4,96 a$ \\
\hline $\mathrm{CV}(\%)$ & 49,36 & 31,27 & 88,47 \\
\hline
\end{tabular}

Áreas de estudo: MN (Mata Nativa) e Sistemas de Produção (ORG 1, ORG 2, CONV 1 e CONV 2). Coeficiente de variação (CV \%). Médias seguidas de mesma letra na coluna não diferem entre si pelo Teste de Tukey a $5 \%$ de significância.

Os atributos microbiológicos não apresentaram diferença significativa entre as áreas (Tabela 4). Em outras condições, para a cultura do cafeeiro sob diferentes sistemas de cultivo, o mesmo foi observado por Guimarães et al. (2017) quando avaliaram que os atributos microbiológicos do solo, no município de Santo Antônio do Jardim (SP).

Os valores de atividade microbiana observados podem ser considerados benéficos para os sistemas estudados, uma vez que maior decomposição de resíduo orgânico se refere à maior disponibilidade de nutrientes às plantas (Medeiros et al., 2019) e estímulo à atividade de outros organismos no solo (Amorim et al., 2020, Balota, 2018). Dessa forma, tem-se que a camada superficial do solo, beneficiada pelo aporte de biomassa da bananeira, pode ter favorecido as condições de temperatura interna, umidade e aeração e, consequentemente,

Faria., V., L., Melloni., R., Melloni., E., G., P. 
propiciaram ambientes protegidos consoantes ao desenvolvimento da população microbiana e sua atividade.

Segundo Balota (2018), a ausência de revolvimento do solo nos sistemas de produção avaliados resulta na maior presença de raízes, aumentando a entrada de substratos carbonados no sistema, via exudatos radiculares. Em áreas sob cultivo de banana, a maior porcentagem das raízes está disposta nas camadas mais superficiais do solo, podendo atingir $\mathrm{o}$ comprimento variável entre $5 \mathrm{~m}$ e $10 \mathrm{~m}$, dependendo da cultivar e das condições do solo (Dantas et al., 2016). Em geral, 70\% das raízes são encontradas a $0,20 \mathrm{~m}$ de profundidade e a 1,50 m do pseudocaule (Simão, 1998).

Quanto à homogeneidade da distribuição do CBM nas áreas cultivadas, pode-se atribuir que as práticas de manejo que não utilizam do revolvimento do solo, aliada aos efeitos da deposição da biomassa da bananeira, proporcionam maior quantidade de $\mathrm{C}$ orgânico para ser imobilizado pela comunidade microbiana do solo, melhorando, assim, a qualidade microbiana do solo (Araújo et al., 2019). Outras pesquisas mostram que a cobertura do solo com resíduo vegetal em sistemas agrícolas estimula positivamente a biomassa microbiana por esses sistemas gerarem microhábitats favoráveis e sítios de refúgio, além do fato dos resíduos vegetais servirem como fonte de energia e nutrientes para os organismos do solo (Guimarães et al., 2017; Pires et al., 2020).

Finalmente, os valores obtidos para $q \mathrm{CO}_{2}$ foram consideravelmente inferiores aos apresentados por Guimarães et al. (2017) quando estes variaram de 15,33 a 30,25 $\mu \mathrm{gCO} 2 / \mu \mathrm{g}$ C. $\mathrm{g}$ de solo seco.dia. Estes resultados evidenciam a ocorrência de maior gasto de energia para a manutenção microbiana, ou seja, em situações estressantes, como o manejo intensivo do solo, os microrganismos precisarão consumir mais substrato para sobrevivência (Pires et al., 2020). Contrariamente, o cultivo orgânico de bananas, avaliado por Aguiar et al. (2013) no município de Umuarama (PR), apresentou os menores valores $q \mathrm{CO}_{2}$ comparativamente ao solo cultivado com palmeiras imperiais que apresentaram valores elevados como indicativos de ecossistemas submetidos a condições de estresse ou de distúrbio causado por diferentes usos ou manejos do solo.

Dessa forma, em observância aos resultados apresentados por estes autores, podese inferir que as áreas avaliadas no presente trabalho apresentam ambientes mais estáveis, com maior densidade microbiana em equilíbrio e baixo estresse microbiológico. Assim, entende-se que houve homogeneidade da qualidade microbiológica do solo, independentemente do sistema, sendo um indicativo de equilíbrio, possivelmente em função das condições ambientais proporcionadas pela qualidade do material orgânico, como a biomassa da bananeira, adicionada ao longo do tempo.

\section{Conclusões}

As práticas culturais e o manejo do solo para a cultura da banana em Gonçalves (MG) contribuem para melhoria da qualidade do solo, em seus aspectos físico, químico e microbiológico, independentemente dos sistemas de produção orgânico ou convencional adotados.

Dessa forma, recomenda-se que, além de manter a cobertura do solo com a biomassa da banana, sejam revistas as práticas de adição de insumos agrícolas nos sistemas de produção convencional.

\section{Agradecimentos}

Os autores agradecem aos produtores de banana de Gonçalves (MG) que, gentilmente, disponibilizaram suas áreas agrícolas para o presente estudo. Agradecem também a Coordenação de Aperfeiçoamento de Pessoal de Nível Superior - Brasil (CAPES) Código de financiamento 001 e a Fundação de Amparo à Pesquisa do Estado de Minas Gerais - Brasil (Fapemig) - Código de Financiamento 001, bem como a Universidade Federal de Itajubá (Unifei) pelo suporte técnico e profissional.

\section{Referências}

Aguiar, D., Lermen, C., Morelli, F., Andrade, L., Pascotto, C.R., Gazim, Z.C., Alberton, O., 2013. Fungos micorrízicos arbusculares, biomassa e atividade microbiana de solo sob banana, braquiária e degradado. Arquivo de 
Ciências Veterinárias e Zoologia da UNIPAR, 16, 137-142. Disponível em: https://www.revistas.unipar.br/index.ph p/veterinaria/article/view/4500/2721.

Acesso em: 08 abr. 2021.

Aires, E.S., Marinho, L.B., Gomes, I.L.S., Silva, J.F.M., Santos, K.C., Andrade, E.A., Araújo, J.F., 2019. Alteração nos atributos físico-hídricos de um Neossolo flúvico sob manejo convencional e orgânico. Water Resources and Irrigation Management 8, 517. Disponível em: https://core.ac.uk/download/pdf/288289946 .pdf. Acesso em: 7 abr. 2021.

Alexandre, C., 2015. Funções, usos e degradação do solo. In: Figueiredo, T. Fonseca, F., Nunes, L. (Org.). Proteção do Solo e combate à desertificação: oportunidade para as regiões transfronteiriças. Bragança: Serviços de Imagem do Instituto Politécnico de Bragança. Disponível em: https://bibliotecadigital.ipb.pt/bitstream/ 10198/12212/3/Protec\%cc\%a7a\%cc\%8 3o\%20do\%20solo.pdf. Acesso em: 7 abr. 2021.

Almeida, U.O., Andrade Neto, R.C., Costa, D.A., Araújo, J.M., Lunz, A.M.P., 2019. Qualidade pós-colheita de banana, cultivar D'Angola, produzida em Rio Branco, Acre. Enciclopédia Biosfera, 16, 1-9. Disponível em:

http://www.conhecer.org.br/enciclop/20 19a/agrar/qualidade\%20pos.pdf. Acesso em: 8 abr. 2021.

Amorim, M.S., Coelho, E.F., Melo, D.M., Lima, D.B., Lima, L.W.F.L., 2019. Cultivo orgânico da bananeira 'BRS Tropical' sob irrigação e uso de cobertura orgânica no solo. Revista Brasileira de Agricultura irrigada 13, 3487-3497. Disponível em: 10.7127/rbai.v13n301085. Acesso em: 7 abr. 2021.

Amorim, S.P.N., Boechat, C.L., Duarte, L.S.L., Oliveira, D.F., Medeiros, J.C., Arauco, A.M.S., 2020. Microbial responses to doses of cover plant straw in cerrado piauiense oxisol. Bioscience Journal, 36, 1146-1155. Disponível em: https://doi.org/10.14393/BJv36n4a2020-47963. Acesso em: 7 abr. 2021.
Amaro, A.A., Fagundes, P.R.S., 2016. Aspectos econômicos e comercialização. In: Ferreira, C.F., Silva, S.O., Amorim, E.P., SantosSerejo, J.A. (Org.). O agronegócio da banana. Brasília: Embrapa, 727-772. Disponível em: https://livimagens.sct.embrapa.br/amostras/ 00084630.pdf. Acesso em: 7 abr. 2021.

Anderson, J.P.E., 1982. Soil respiration. In: Page, A.L., Miller, R.H., Keeney, D.R. Methods of soil analysis: Chemical and microbiological properties. 2 ed. Madison, Soil Science Society of America/American Society of Agronomy. Part 2. p.831-845.

Anderson, T.H., Domsch, K.H., 1993. The metabolic quotient for $\mathrm{CO} 2(\mathrm{qCO} 2)$ as a specific activity parameter to assess the effects on environmental conditions, such as $\mathrm{pH}$, on the biomass of forest soils. Soil Biology and Biochemistry 25, 393-395. Disponível em: https://doi.org/10.1016/00380717(93)90140-7. Acesso em: 7 abr. 2021.

Araujo, T.S., Gallo, A.S., Araujo, F.S., Santos, L.C., Guimarães, N.F., Silva, R.F., 2019. Biomassa e atividade microbiana em solo cultivado com milho consorciado com leguminosas de cobertura. Revista de Ciências Agrárias, 42, 347-357. Disponível em: https://doi.org/10.19084/rca.15433. Acesso em: 7 abr. 2021.

Assunção, S.A., Pereira, M.G., Rosset, J.S., Berbara, R.L.L., García, A.C., 2019. Carbon input and the structural quality of soil organic matter as a function of agricultural management in a tropical climate region of Brazil. Science of the total environment 658, 901-911. Disponível em: https://doi.org/10.1016/j.scitotenv.2018.12. 271. Acesso em: 7 abr. 2021.

Balota, E.L., 2018. Manejo e qualidade biológica do solo. Londrina: EDMidiograf, 280p.

Barbosa, T.C., Costa, N.M.G.B., Santos, D.B., Machado, M.S., Marques Filho, F., 2020. Qualidade física do solo em áreas sob manejo agroecológico e convencional. Brazilian Journal of Development 6, 4889948909.

Disponível

em: 
https://doi.org/10.34117/bjdv6n7-511.

Acesso em: 7 abr. 2021.

Borges, A.L., Silva, J.T.A., Oliveira, A.M.G., D'Oliveira, P.S., 2016. Nutrição e adubação. In: Ferreira, C.F., Silva, S.O., Amorim, E.P., Santos-Serejo, J.A. (Org.). O agronegócio da banana. Brasília: Embrapa, p. 331-398. Disponível em: https://livimagens.sct.embrapa.br/amostras/ 00084630.pdf. Acesso em: 7 abr. 2021.

Borges, A.L., Souza, L.S., 2009. Atributos físicos e químicos de solos cultivados com bananeira, sob irrigação, no Projeto Formoso, Bom Jesus da Lapa, Bahia. Cruz das Almas: Embrapa Mandioca e Fruticultura Tropical, 33 p. Disponível em: https://core.ac.uk/download/pdf/154289 28.pdf. Acesso em: 7 abr. 2021.

Borges, A.L., Souza, L.S., Melo, F.C.M., 2018. Índice de qualidade de solos cultivados com bananeira nas regiões oeste da Bahia e no norte de Minas Gerais. Cruz das Almas: Embrapa Mandioca e Fruticultura, 29 p. Disponível em: https://ainfo.cnptia.embrapa.br/digital/bitstr eam/item/183085/1/BoletimPesquisa94AnaLucia-.pdf. Acesso em: 7 abr. 2021.

Cesaril, C.E., Oliveira Filho, L.C.I., Santos, J.C., Rosa, M.G., 2019. Fauna edáfica em sistemas de produção de banana no Sul de Santa Catarina. Agronomia (Agronomy) 14, 1-12. Disponível em: 10.5039/agraria.v14i1a5613. Acesso em: 7 abr. 2021.

Dantas, A.C.V.L., Almeida, W.A.B., Dantas, J. L.L., Alves, E.J., 2016. Estrutura da planta. In: Ferreira, C.F., Silva, S.O., Amorim, E.P., Santos-Serejo, J.A. (Org.). O agronegócio da banana. Brasília: Embrapa, 29-44. Disponível em: https://ainfo.cnptia.embrapa.br/digital/bitstr eam/item/183085/1/BoletimPesquisa94AnaLucia-.pdf. Acesso em: 7 abr. 2021.

EMBRAPA. Empresa Brasileira de Pesquisa Agropecuária, 1997. Manual de métodos de análise de solo. Rio de Janeiro. Disponível em:

https://www.agencia.cnptia.embrapa.br/Rep ositorio/Manual+de+Metodos_000fzvhotqk 02wx5ok0q43a0ram31wtr.pdf. Acesso em: 1 fev. 2020.

Fagundes, M.O., Reis, D.A., Portella, R.B., Perina, F.J., Bogiani, J.C., 2019. Qualidade de um latossolo sob plantio convencional e sistema palntio direto no cerrado baiano, Brasil. Revista Ibero-Americana de Ciências Ambientais 10, 281-297. Disponível em: 10.6008/CBPC2179-6858.2019.003.0024.

Acesso em: 7 abr. 2021.

FAO. Organização das Nações Unidas para a Alimentação e a Agricultura, 2017. Disponível em: http://www.fao.org/news/archive/news-bydate/2017/pt/. Acesso em: 9 fev. 2020.

FAO. Organização das Nações Unidas para a Alimentação e a Agricultura, 2019. FAO prevê fortes perspectivas de crescimento para produção global e comércio de frutas tropicais. 2019. Rio de Janeiro. Disponível em:

http://www.fao.org/brasil/noticias/detailevents/en/c/1193684/. Acesso em: 9 out. 2019.

Ferreira, A.S., Camargo, F.A.O., Vidor, C., 1999. Utilização de microondas na avaliação da biomassa microbiana do solo. Revista Brasileira de Ciência do Solo 23, 991-996. Disponível em: https://www.scielo.br/pdf/rbcs/v23n4/26.pd f. Acesso em: 7 abr. 2021.

Ferreira, D.F., 2011. Sisvar: a computer statistical analysis system. Ciência e Agrotecnologia 35. Disponível em: https://doi.org/10.1590/S141370542011000600001. Acesso em: 7 abr. 2021.

Freitas, L., Oliveira, I.A., Silva, L.S., Frare, J.C.V., Filla, V.A., Gomes, R.P., 2017. Indicadores da qualidade química e física do solo sob diferentes sistemas de manejo. Unimar Ciências 26, 08-25. Disponível em: http://ojs.unimar.br/index.php/ciencias/artic le/view/511/278. Acesso em: 7 abr. 2021.

Guimarães, N.F., Gallo, A.S., Fontanetti, A., Menegin, S.P., Souza, M.D.B., Morinigo, K.P. G., Silva, R.F., 2017. Biomassa e atividade microbiana do solo em diferentes sistemas de cultivo do cafeeiro. Revista de 
Ciências Agrárias 40, 34-44. Disponível em: https://doi.org/10.19084/RCA16041.

Acesso em: 7 abr. 2021.

IBGE. Instituto Brasileiro de Geografia e Estatística, 2019. Disponível em: https://cidades.ibge.gov.br/brasil/pesquisa/1 5/11863. Acesso em: 15 fev. 2020.

Jesus, M.C., Brito, A.S., Silva, M.O., Teixeira, S.S., Carvalho, W.D., 2017. Permeabilidade ao ar e porosidade de solos a região semiárida. Revista Engenharia na Agricultura 25, 230-239. Disponível em: https://doi.org/10.13083/reveng.v25i3.739.

Acesso em: 7 abr. 2021.

Li, P., Shi, K., Wang, Y., Kong, D., Liu, T., Jiao, J., Liu, M., Li, H., Hu, F., 2019. Soil quality assessment of wheat-maize cropping system with different productivities in China: Establishing a minimum data set 190, 31-40. Disponível em: https://doi.org/10.1016/j.still.2019.02.019.

Acesso em: 7 abr. 2021.

Loss, A., Santos Junior, E., Schmitz, D., Veiga, M., Kurtz, C., Comin, J.J., 2017. Atributos físicos do solo em cultivo de cebola sob sistemas de plantio direto e preparo convencional. Revista Colombiana de Ciencias Hortícolas, 11, 105-113. Disponível em: http://dx.doi.org/10.17584/rcch.2017v1 1i1.6144. Acesso em: 7 abr. 2021.

Medeiros, E.V., Silva, A.O., Duda, G.P., Santos, U.J., Souza Junior, A.J., 2019. The combination of Arachis pintoi green manure and natural phosphate improves maize growth, soil microbial community structure and enzymatic activities. Plant and soil, 435, 175-185. Disponível em: https://doi.org/10.1007/s11104-018-3887-z. Acesso em: 7 abr. 2021.

Melo, V.F., Silva, D.T., Evald, A., Rocha, P.R.R., 2017. Qualidade química e biológica do solo em diferentes sistemas de uso em ambiente se savana. Revista Agroambiente On-line 11, 101-110. Disponível em: https://doi.org/10.18227/19828470ragro.v11i2.3850. Acesso em: 7 abr. 2021.

Faria., V., L., Melloni., R., Melloni., E., G., P.
Mioto, L.C., Araújo, M.A., Seron, C.C., Lavanholi, R., Batista, M.A., Ushiwata, S.Y., 2020. Resistência mecânica do solo à penetração avaliada em área de segundo ano de implantação da cultura da mandioca (Manihot esculenta CRANTZ) 6, 46014620. Disponível em: $10.34117 / \mathrm{bjdv} 6 \mathrm{n} 1-$ 330. Acesso em: 7 abr. 2021.

MMA. Ministério do Meio Ambiente, 2019. Disponível em: http://www.sema.df.gov.br/. Acesso em: 18 dez. 2020.

Morais, E.R.C., Oliveira, A.A.S., Maia, C., 2014. Qualidade do solo cultivado com banana irrigada e sua relação com áreas de caatinga. Revista Brasileira de Engenharia Agrícola e Ambiental 18, 887-891. Disponível em: http://dx.doi.org/10.1590/18071929/agriambi.v18n09p887-891. Acesso em: 7 abr. 2021.

Moura Filho, E.R., Macedo, L.P.M., Silva, A.R.S., 2015. Levantamento fitossociológico de plantas daninhas em cultivo de banana irrigada. Holos 2, 92-97. Disponível em: https://doi.org/10.15628/holos.2015.10 06. Acesso em: 7 abr. 2021.

Olivares, B.O., Araya-Alman, M., AcevedoOpazo, C., Rey, J.C., Cañete-Salinas, P., Kurina, F.G., Balzarini, M., Lobo, D., Navas-Cortés, J., Landa, B.B., Gómez, J.A., 2020. Relationship between soil properties and banana productivity in the two main cultivation áreas in Venezuela. Journal of Soil Science and Plant Nutrition, 20, 25122524. Disponível em: https://doi.org/10.1007/s42729-020-003178. Acesso em: 7 abr. 2021.

Oliveira, B.A., Campos, L.P., Matias, S.S.R., Silva, T.S., Gualberto, A.V.S., 2021. Spatiality of soil chemical attributes in a banana cultivation área in West Bahia. Revista Caatinga, 34, 177-188. Disponível em:

https://periodicos.ufersa.edu.br/index.ph p/caatinga/article/view/9192/10570.

Acesso em: 8 abr. 2021.

Pires, M.F.M., Medeiros, J.C., Souza, H.A., Rosa, J.D., Boechat, C.L., Mafra, A.L., Nolêto, K.C., 2020. Conservation system 
improves soil microbial quality and increases soybean yield in the Northeastern Cerrado. Bragantia, 79, 474-486. Disponível em: $\quad$ https://doi.org/10.1590/16784499.20200117. Acesso em: 7 abr. 2021.

Prakash, S., Shyam, R., Prasad, J., 2019. Impact of conservation agriculture on growth, yield, and quality of banana (musa spp.) Cv. Grand Naine. Current Journal of Applied Science and Technology, 37, 1-6. Disponível em: $\quad 10.9734 /$ cjast/2019/v37i630329.

Acesso em: 7 abr. 2021.

Ribeiro, P.L., Bamberg, A.L., Kunde, R.J., Stöcker, C.M., Monteiro, A.B., Martinazzo, R., 2018. Condições físicas de Chernossolos cultivados com soja na bacia hidrográfica do Rio Santa Maria, RS. Revista Brasileira de Ciências Agrárias (Agrária) 13, e5560. Disponível em: 10.5039/agraria.v13i3a5560. Acesso em: 7 abr. 2021.

Rosa, V.A., Soares Neto, J.P., Nunes, H.B., Calisto, K.S., Cruz, J.N., 2019. Atributos físicos e estoque de carbono em sistemas agroflorestais nos Cerrados do oeste da Bahia. Revista Brasileira de Geografia Física, 12, 2660-2671. Disponível em: https://doi.org/10.26848/rbgf.v12.7.p2 660-2671. Acesso em: 7 abr. 2021.

Rossi, C.Q., Pereira, M.G., Moura, O.V.T., Almeida, A.P.C., 2016. Vias de formação, estabilidade e características químicas de agregados em solos sob sistemas de manejo agroecológicos. Pesquisa Agropecuária Brasileira 51, 1677-1685. Disponível em: https://doi.org/10.1590/s0100-

204x2016000700068. Acesso em: 7 abr. 2021.

Sales, R.P., Portugal, A.F., Moreira, J.A.A., Kondo, M.K., Pegoraro, R.F., 2016. Qualidade física de um Latossolo sob plantio direto e preparo convencional no semiárido. Revista Ciência Agronômica 47, 429-438. Disponível em: 10.5935/18066690.20160052. Acesso em: 7 abr. 2021.

Schembergue, A., Cunha, D.A., Carlos, S.M., Pires, M.V., Faria, R.M., 2017. Sistemas agroflorestais como estratégia de adaptação aos desafios das mudanças climáticas no Brasil. Revista de Economia e Sociologia Rural 55, 9-30. Disponível em: https://doi.org/10.1590/1234-5678180694790550101. Acesso em: 7 abr. 2021.

Shapiro, S.S., Wilk, M.B., 1965. An analysis of variance test for normality (complete sample). Biometrika 52, 591-611. Disponível em: https://doi.org/10.2307/2333709. Acesso em: 7 abr. 2021.

Silva, J.T.A., Pacheco, D.D., Costa, E.L., 2007. Atributos químicos e físicos de solos cultivados com bananeira 'Prata-Anã' (AAB), em três níveis de produtividade, no norte de Minas Gerais. Revista Brasileira de Fruticultura 29, 102-106. Disponível em: http://dx.doi.org/10.1590/S0100-

29452007000100022. Acesso em: 7 abr. 2021.

SIMÃO, S. Tratado de fruticultura. Piracicaba: Fealq, 1998. 760 p.

Souza, K.R., Melloni, R., Ferreira, G.M.R., Souza, S.M.P., Rezende, J.T., 2016. Proposta da metodologia RAQS para avaliação visual da qualidade do solo. Revista Brasileira de Geografia Física, 9, 1815-1824. Disponível em: https://doi.org/10.26848/rbgf.v9.6.p18151824. Acesso em: 7 abr. 2021.

UCB. Unidades de Conservação no Brasil, 2013. Disponível em: https://uc.socioambiental.org/. Acesso em: 13 set. 2019.

Yang, X., Tsibart, A., Nam, H., Hur, J., ElNaggar, A., Tack, F.M.G., Wang, C.H., Lee, Y.H., Tsang, D.C.W., Ok, Y.S., 2019. Effect of gasification biochar application on soil quality: Trace metal behavior, microbial community, and soil dissolved organic matter. Journal of Hazardous Materials, 365, 684-694. Disponível em: https://doi.org/10.1016/j.jhazmat.2018.11.0 42. Acesso em: 7 abr. 2021. 